\title{
Ginkgetin aglycone attenuates neuroinflammation and neuronal injury in the rats with ischemic stroke by modulating STAT3/JAK2/SIRT1
}

\author{
Bing $\mathrm{Xu}^{1}$, Xin $\mathrm{He}^{2}$, Yi Sui ${ }^{1}, \mathrm{Xu}$ Wang $^{1}$, Xia Wang ${ }^{1}$, Li Ren ${ }^{1}$, Yun-Xin Zhai ${ }^{1}$ \\ ${ }^{1}$ Department of Neurology, Shenyang First Hospital, Shenyang, ${ }^{2}$ Department of Neurology, Anyang District Hospital, Puyang, China
}

\begin{abstract}
The present investigation evaluates the protective effect of Ginkgetin aglycone (GA) against ischemic stroke-induced neuronal injury. Ischemic stroke was produced by the middle cerebral artery occlusion (MCAO) model and animals were a group that received GA 100 and $200 \mathrm{mg} / \mathrm{kg}$, i.p. five days before the induction of MCAO. The effect of GA against stroke was determined by estimating the neurological deficit score and brain water content was also observed. Moreover terminal deoxynucleotidyl transferase dUTP nick end labelling (TUNEL) assay was done for determining the neuronal apoptosis and Western blot assay also performed for estimating the expression of several proteins. Results of the study suggest that the neurological deficit score and brain water content was found to be lower in the GA treated group than the ischemia/reperfusion (I/R) group of rats. Moreover the number of TUNEL positive cells was found to be lower in the GA treated group than in the I/R group of rats. There was a significant $(p<0.01)$ decrease in the oxidative stress parameters and cytokine in the tissue homogenate of the GA treated group compared to the I/R group of rats. Further treatment with GA attenuates altered expression of phosphatidylinositol-4,5-bisphosphate 3-kinase (PI3K), protein kinase B (Akt), B-cell lymphoma 2 (BCl-2), signal transducer and activator of transcription 3 (STAT3), nuclear factor kappa light chain enhancer of activated B cells (NF-KB), toll-like receptor 4 (TLR-4), Janus kinase 2 (JAK-2) and sirtuin-1 (SIRT-1) protein in the brain tissues of stroke rats. In conclusion, data of the report reveal that treatment with Ginkgetin aglycone protects the neuronal injury against stroke in rats by reducing oxidative stress and inflammation.
\end{abstract}

Key words: ginkgetin aglycone, ischemic stroke, neuroinflammation, neuronal injury, oxidative stress.

\section{Introduction}

Stroke is one of the major causes of mortality throughout the world and several complications are associated with this such as coma, hemiplegia and death. Ischemic stroke often occurs due to involvement of the middle cerebral artery [14]. Cerebral ischemia leads to progression of neuronal injury by cellular necrosis, apoptosis and oedema [19]. Moreover, reperfusion after ischemia also alleviates the production of reactive oxygen species (ROS), neuronal apoptosis and inflammation which enhances the cerebral injury [4]. The literature reveals that many pathways are involved in I/R induced cerebral injury such as activation of JAK2/STAT3 signalling pathway reported to protect cerebral injury [20]. Moreover Sirt-1 is a histone deacetylases enzyme which protects the cerebral ischemia induced brain injury [10]. Conventional therapy available for the management

\section{Communicating author}

Bing Xu, PhD, Department of Neurology, Shenyang First Hospital, No. 67 Qingquan Road, 110042, Shenyang, China,

e-mail: BeverlygMitchellyn@yahoo.com 
of stroke has several limitations and thus there is a need to explore the novel therapeutic agents.

There are several molecules from the herbal sources used for the management of several disorders including the stroke. Ginkgo biloba was traditionally used in China for the management of several disorders due to its antioxidant activity [7]. Ginkgo biloba was reported to contain terpenoids and flavonoids like chemical constituents which possess strong anti-inflammatory, antioxidant and free radical scavenging activity [16]. GA is reported for protecting $I / R$ induced kidney injury on the basis of its anti-inflammatory and antioxidant properties [1]. GA reduces the inflammatory pathway by inhibiting nuclear factor kappa light chain enhancer of activated $B$ cells (NF- $\kappa B$ ) signalling and activates Sirt-1 enzyme and thereby modulates the apoptosis [23]. Thus, the present report evaluates the cerebral protective effect of Ginkgetin aglycone against the ischemic stroke induced neuronal injury rat model.

\section{Material and methods \\ Animals}

Male Wistar rats were procured from the Peking University Health Science Center, China. All the animals were kept under the standard conditions such as $12 \mathrm{~h}$ light and dark cycle at $24 \pm 3^{\circ} \mathrm{C}$ for $60 \pm 5 \%$ of humidity as per the guideline and acclimatized for the period of one week before the start of the experimentation. All the protocols of this study were approved by the Institutional Animal Care and Use Committee of Shenyang First Hospital, China (IACUC/ SFH/2017/02) and the study followed the guidelines of the Association for the Assessment and Accreditation of Laboratory Animal Care International (AAALAC) for experimentation and animal use.

\section{Chemicals}

Ginkgetin aglycone was procured from Shanghai Yuanye Biotechnology Co., Ltd, China and the TUNEL system kit was purchased from Promega (Beijing) Biotech Co., Ltd, China. ELISA kits of NF- $\kappa B$, interleukin $1 \beta$ (IL-1 $\beta)$, tumour necrosis factor $\alpha$ (TNF- $\alpha)$, SOD and MDA were purchased from Gene Company Limited, China. Antibodies such as PI3K, Akt, Bcl-2, STAT3, NF-KB, TLR-4, JAK2, SIRT-1, glyceraldehyde-3-phosphate dehydrogenase (GADPH) and $\beta$-actin were procured from Thermo Fisher Scientific Ltd., USA.

\section{Experimentation}

The ischemic stroke was produced by the middle cerebral artery occlusion (MCAO) model as per the previous study [18]. All the animals were anesthetized by injecting a $350 \mathrm{mg} / \mathrm{kg}$ dose of chloral hydrate intraperitoneally and monofilament nylon suture was inserted with a heat-rounded tip through the internal carotid artery for the production of MCAO. Further reperfusion was produced by withdrawing the monofilament 30 min after the induction of ischemia. In the Sham group, surgery was performed in the same way without production of MACO. The heating blanket and lamp was used to maintain the temperature at $37^{\circ} \mathrm{C}$ during the period of surgery.

All the animals were separated into 4 different groups such as the Sham operated group, I/R group in which ischemia was induced by MCAO; Ginkgetin aglycone 100 and $200 \mathrm{mg} / \mathrm{kg}$ treated group which receives GA 100 and $200 \mathrm{mg} / \mathrm{kg}$ i.p. five days before the induction of MCAO.

\section{Determination of the neurological function}

The neurological deficit score was determined in each animal as per the previous study [17]. The neurological function was scored as per the pattern given below such as no motor deficits - 0; weakness of forelimb -1 ; circling to the contralateral side -2 ; on the affected side unable to bear the weight -3 ; locomotor activity not spontaneous -4 .

\section{Determination of water content in the brain}

Water content in the brain was estimated as per the previous method [12]. All the animals were sacrificed by cervical dislocation and brain tissue was isolated from each animal. Weight of wet brain tissue was observed and further white and red brain tissue was desiccated until getting constant weight for 2 days at $105^{\circ} \mathrm{C}$. Water content in the brain was determined using the following formula:

Water content: (wet weight - dried weight)/wet weight $\times 100 \%$

\section{TUNEL assay}

TUNEL assay was performed for the evaluation of neuronal apoptosis. All the rats were sacrificed by cervical dislocation and the brain was isolated, fur- 
ther hippocampi were separated from it. The hippocampus tissue was dehydrated and further seeded into paraffin. Furthermore, microtome was used to section into $6 \mathrm{~mm}$ thickness of about $200 \mu \mathrm{m}$ apart. Promega's TUNEL system kit was used to estimate the neuronal apoptosis as per the instructions given by the manufacturer. The estimated number of apoptotic cells was determined using NIS-Elements BR imaging processing and analysis software.

\section{Determination of biochemical parameters}

ELISA kits were used to determine the concentration of mediators of inflammation such as NF- $\mathrm{KB}$, IL-1 $\beta$ and TNF- $\alpha$ in the brain tissues of stroke neuronal injured rats. Moreover, parameters of oxidative stress such as the level of MDA and activity of SOD were estimated in the brain tissues using the ELISA kit.

\section{Western blot assay}

Brain tissues of the injured location were separated out and tissue lysis was done using lysis buffer. Supernatant from the lysed tissue was separated out by centrifuging the lysate for the period of $5 \mathrm{~min}$ at 10000 RPM. Later, sodium dodecyl sulfate-polyacrylamide gel electrophoresis was used to separate the total protein and then separated protein was filtered on nitrocellulose membrane. Further, membrane was incubated with $\mathrm{I}^{\mathrm{ry}}$ antibodies like PI3K (1:500), Akt (1:100), Bcl-2 (1:500), STAT3 (1:100), NF-אB (1: 500), TLR-4 (1:100), JAK2 (1:100), SIRT-1 $(1: 100), \operatorname{GADPH}(1: 100)$ and $\beta$-actin $(1: 100)$

A

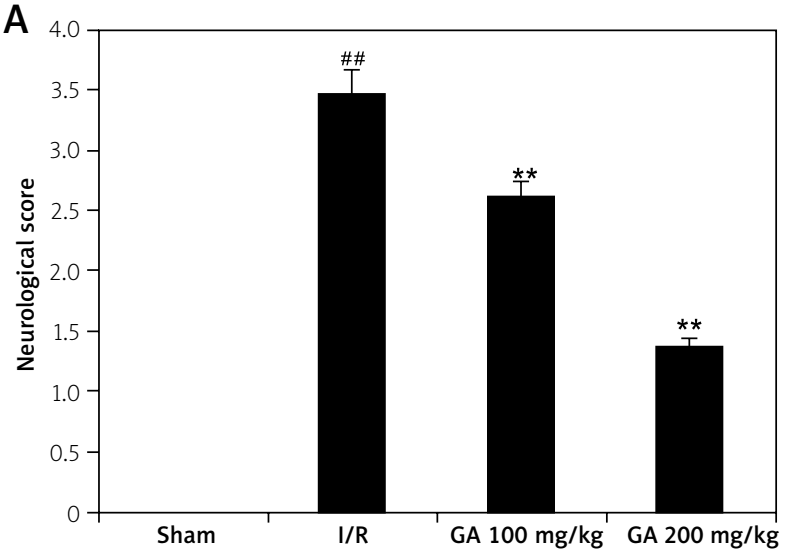

for overnight at $4^{\circ} \mathrm{C}$. Then, the horseradish peroxidase-conjugated secondary antibody was used to incubate for the period of $60 \mathrm{~min}$ at room temperature with total protein. Image I software was used to estimate the band density.

\section{Statistical analysis}

All data were expressed as mean $\pm \operatorname{SEM}(n=10)$. The statistical analysis was performed using one way ANOVA. Post-hoc comparison of means was carried out by Dunnett's post-hoc test (Graphpad prism 6.1., CA, USA) multiple comparisons. The level of statistical significance was set at $p<0.05$.

\section{Results}

\section{Ginkgetin aglycone ameliorates the neurological score and water content}

Figure 1 shows the effect of GA on the neurological function and water content in the brain tissues of the ischemic stroke rat model. The neurological function was determined by estimating the neurological score and there was a significant enhancement of the neurological score in I/R rats compared to the Sham operated group. However, treatment with GA attenuates the increased neurological score in ischemic stroke rats. The percentage of water content in the brain of the I/R group was enhanced compared to the Sham operated group. It was observed that treatment with GA decreases the water content in the brain compared to the I/R group of rats.

B

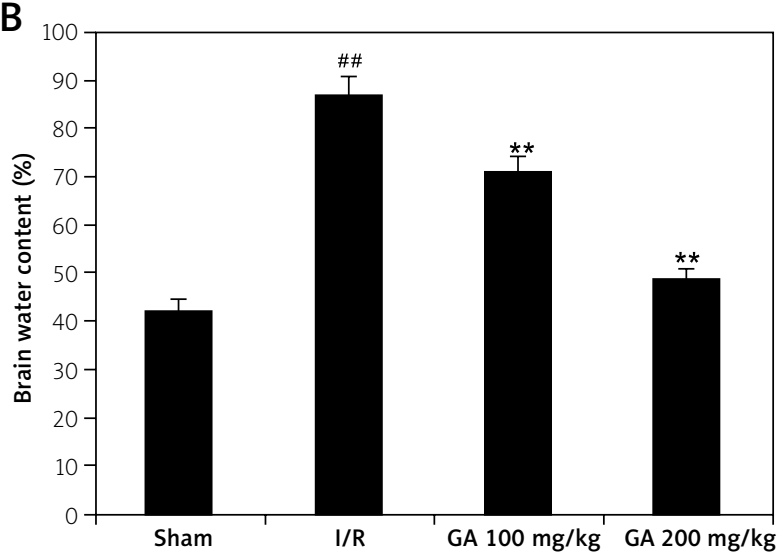

Data expressed as mean $\pm \operatorname{SEM}(n=10)$

${ }^{\# \#} p<0.01$ compared to the Sham group; ${ }^{* *} p<0.01$ compared to the I/R group

Fig. 1. Ginkgetin aglycone ameliorates the neurological score and brain water content in the ischemic stroke rat model. 


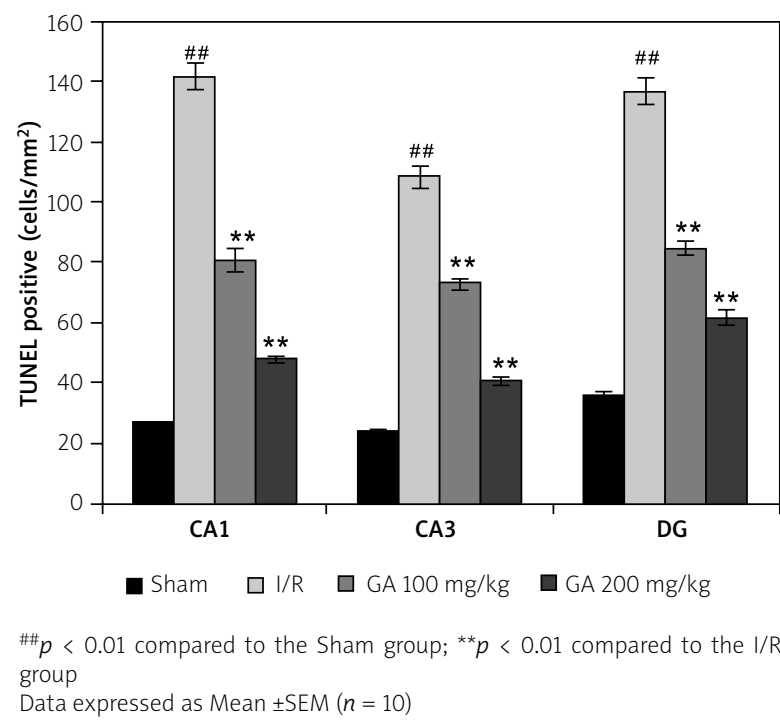

Fig. 2. Ginkgetin aglycone ameliorates the number of TUNEL positive cells in the brain tissues of the ischemic stroke rat model.

\section{Ginkgetin aglycone ameliorates neuronal apoptosis}

TUNEL staining was done to observe neuronal apoptosis in the ischemic stroke rat (Fig. 2). There was a significant increase in the number of TUNEL positive cells in the different regions such as CA1, CA3 and DG of brains of the I/R group compared to the Sham operated group of rats. It was observed that the number of TUNEL positive cells was reduced in the brain tissues of GA treated rats compared to the I/R group of rats.

\section{Ginkgetin aglycone ameliorates pro-inflammatory cytokines}

In ischemic stroke and GA treated rats, the level of cytokines in the brain tissues was estimated and data of the study suggest that the level of NF-KB, TNF- $\alpha$ and IL-1 $\beta$ was significantly enhanced in the I/R group compared to the Sham operated group of rats. However, GA treated rats show that the level of cytokines in the brain tissue was reduced significantly compared to the I/R group of rats (Fig. 3).

\section{Ginkgetin aglycone ameliorates the parameters of oxidative stress}

Figure 4 shows the effect of GA on the level of MDA and activity of SOD in the tissues homogenate

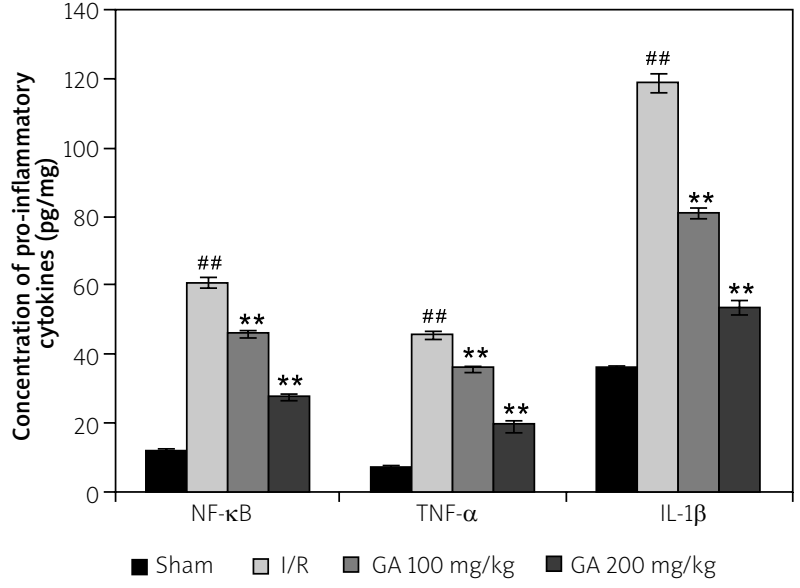

$\#$ \#\# 0.01 compared to the Sham group; ${ }^{* *} p<0.01$ compared to the I/R group

Data expressed as Mean $\pm \operatorname{SEM}(n=10)$

Fig. 3. Ginkgetin aglycone ameliorates pro-inflammatory cytokines in the brain tissues of the ischemic stroke rat model.

of the ischemic stroke rat. It was observed that the level of MDA increases and activity of SOD is reduced in the brain tissues of the I/R group compared to the Sham operated group of rats. There was a decrease in the level of MDA and improvement in the activity of SOD enzyme in the brain tissues of the GA treated group compared to the I/R group of rats.

\section{Ginkgetin aglycone ameliorates PI3K/NF-KB/TLR-4 pathway}

The effect of GA on the PI3K/NF-KB/TLR-4 inflammatory pathway was assessed in the tissue homogenate of ischemic stroke rats (Fig. 5). Expression of Akt and PI3K proteins was reduced and so was expression of NF- $\kappa B$ and TLR-4 in the brain tissue homogenate of the I/R group compared to the Sham operated group of rats. However, PI3K/NF-KB/TLR-4 inflammatory pathway was found to be attenuated in the brain tissues of the GA treated group compared to the I/R group of rats.

\section{Ginkgetin aglycone ameliorates STAT-3/JAK-2/Sirt-1 pathway}

Figure 6 shows the effect of GA on the expression of STAT-3, JAK-2 and SIRT-1 protein in the brain tissues of ischemic stroke rats. Data of investigation reveal that the expression of STAT-3, JAK-2 and SIRT-1 protein was enhanced in the brain tissues of the I/R 

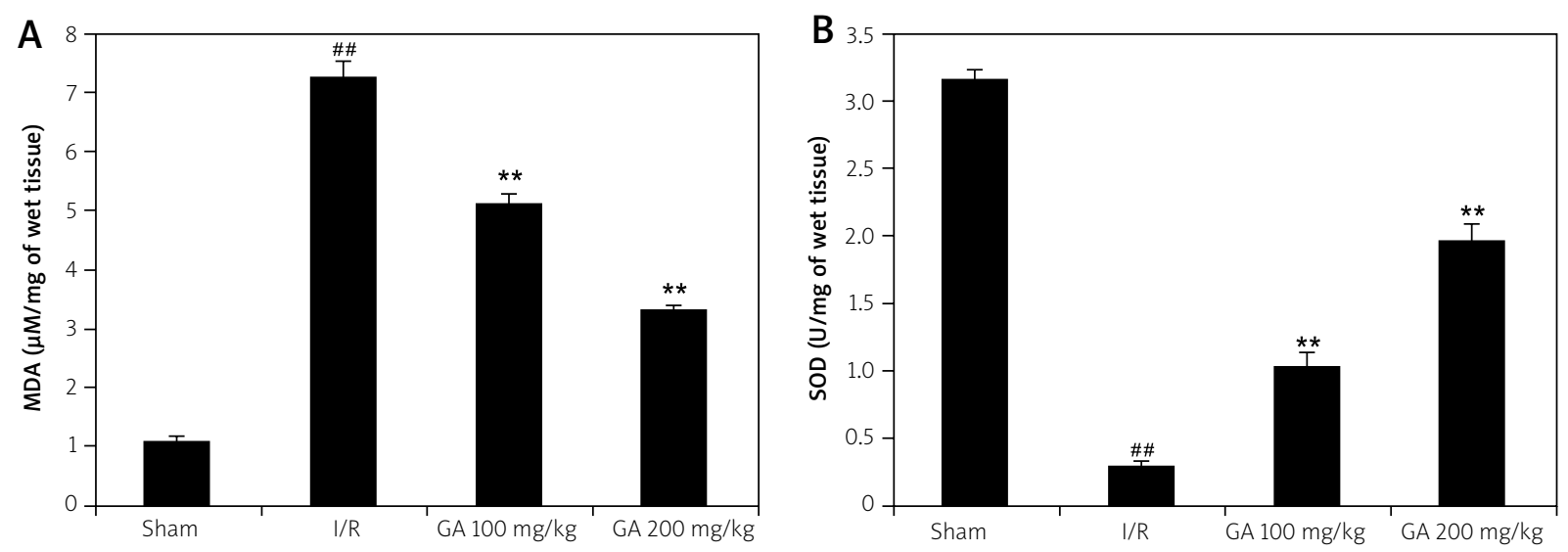

$\#$ \# $p<0.01$ compared to the Sham group; ${ }^{* *} p<0.01$ compared to the I/R group Data expressed as Mean \pm SEM $(n=10)$

Fig. 4. Ginkgetin aglycone ameliorates parameters of oxidative stress in the brain tissues of the ischemic stroke rat model.
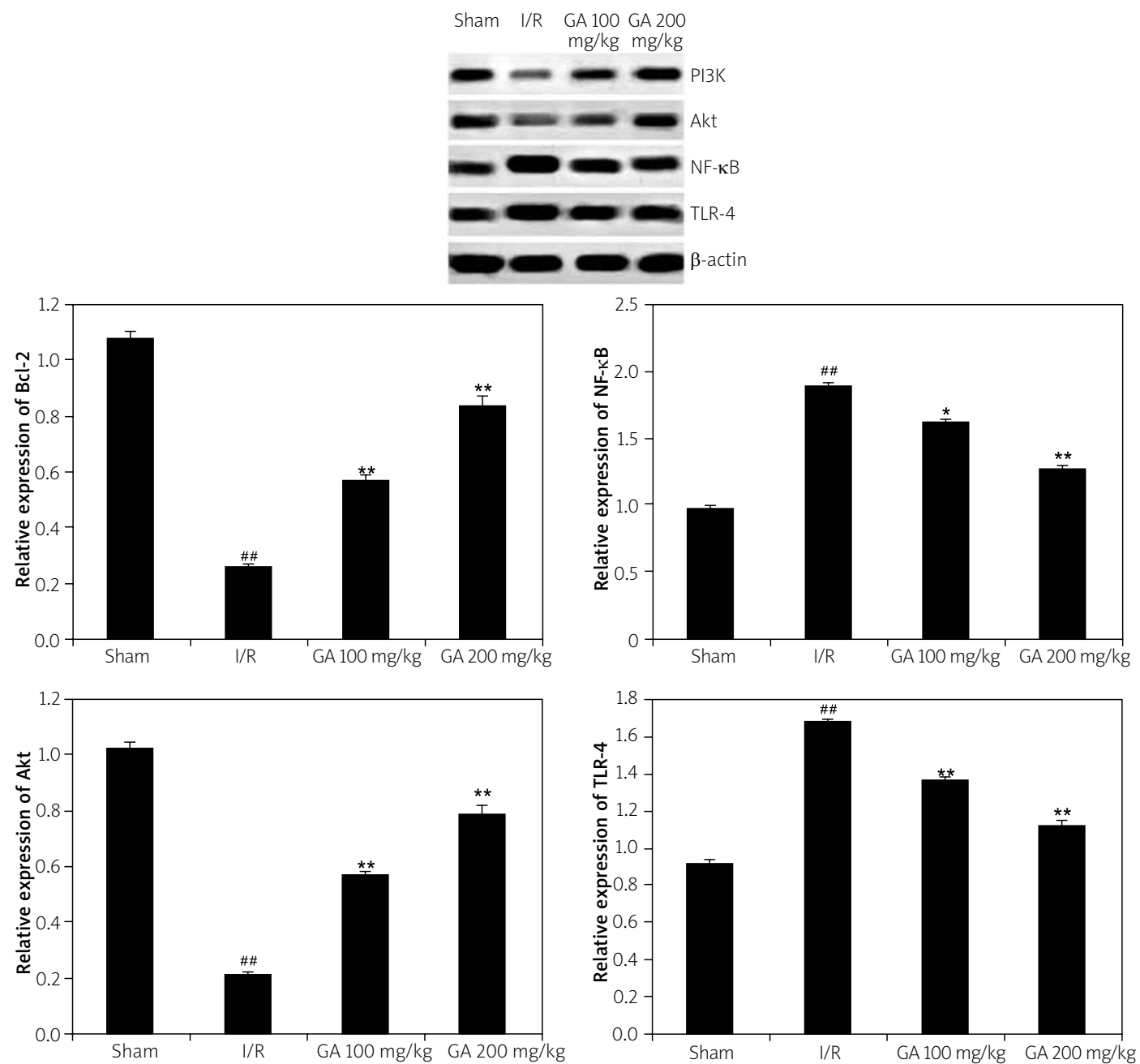

Data expressed as Mean \pm SEM $(n=10)$

$\# \# p<0.01$ compared to the Sham group; ${ }^{*} p<0.05,{ }^{* *} p<0.01$ compared to the I/R group

Fig. 5. Ginkgetin aglycone ameliorates the relative expression of PI3K, Akt, NF- $\kappa B$ and TLR-4 protein in the brain tissues of the ischemic stroke rat model. 

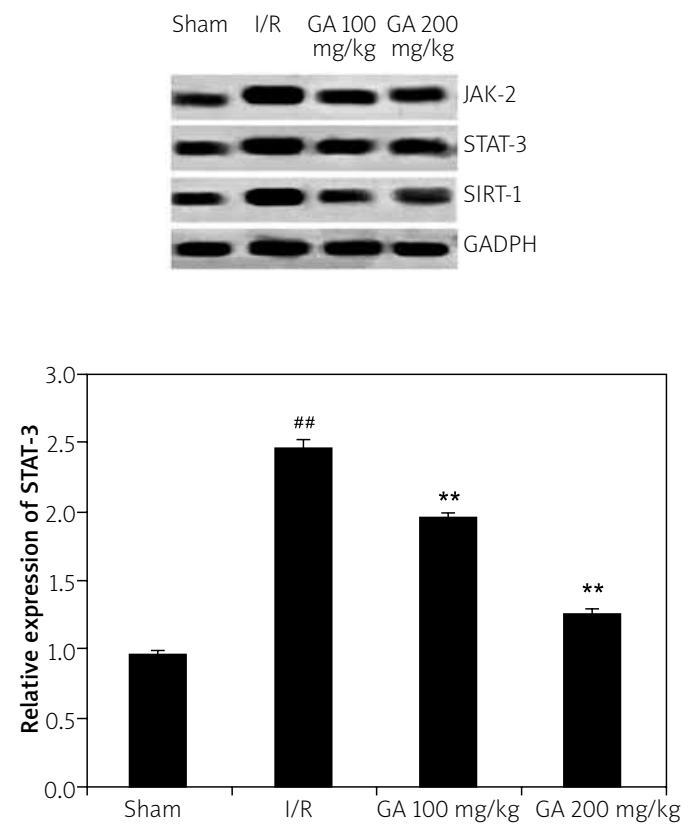

Data expressed as Mean $\pm \operatorname{SEM}(n=10)$

$\# p<0.01$ compared to the Sham group; ${ }^{* \star} p<0.01$ compared to the I/R group
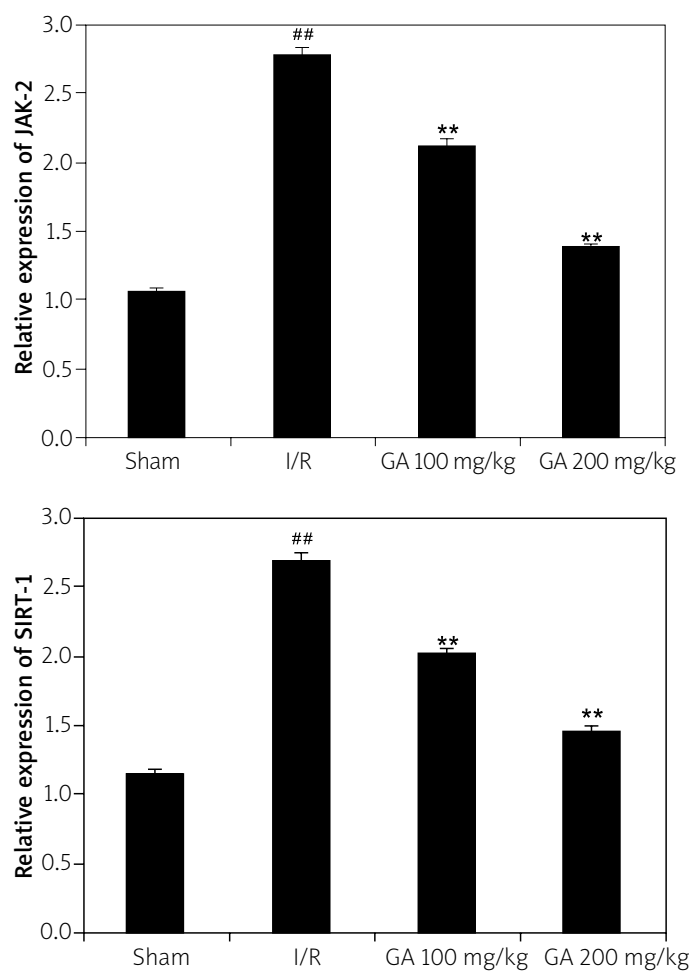

Fig. 6. Ginkgetin aglycone ameliorates the relative expression of STAT-3, JAK-2 and SIRT-1 protein in the brain tissues of the ischemic stroke rat model.

group compared to the Sham operated group of rats. However, treatment with GA attenuates the altered expression of STAT-3, JAK-2 and SIRT-1 protein in the brain tissues of ischemic stroke rats.

\section{Discussion}

Ischemic stroke is one of the major causes of death throughout the globe and its management still remains a challenge for medicine. In the last few decades, alternative medicine has shown a significant role and efficacy against the management of several disorders including stroke. Thus, this investigation evaluates the protective effect of Ginkgetin aglycone against ischemic stroke induced neuronal injury. The effect of GA against stroke was determined by estimating the neurological deficit score and brain water content was also observed. Moreover, TUNEL assay was done for determining the neuronal apoptosis and Western blot assay was also performed for estimating the expression of several proteins.
It is well documented that ischemic stroke results into neuronal inflammation and injury. There are several factors that contribute in the pathophysiology of neuronal injury in the stroke such as oxidative stress and level of inflammatory cytokines [9]. The literature reveals that generation of reactive oxygen species is enhanced in the stroke condition, which further leads to an increase in the production of $\mathrm{H}_{2} \mathrm{O}_{2}$ [8]. $\mathrm{H}_{2} \mathrm{O}_{2}$ reported to be toxic for cells by altering the cell signalling pathways [11]. Moreover, the stroke leads to activation of granulocytes which enhances the level of inflammatory cytokines in the ischemic tissues. Inflammation causes the imbalance between the endogenous antioxidant molecules and production of oxidative stress in the cell [2]. Drugs used for the management of neuronal injury were reported to decrease the production of ROS, oxidative stress and inflammation [15]. Data of our study reveal that treatment with GA ameliorates the oxidative stress and inflammation in the brain tissue of stroke rats. 
There are several pathways involved in the neuronal inflammation including $\mathrm{PI} 3 \mathrm{~K} / \mathrm{NF}-\mathrm{KB} / \mathrm{TLR}-4$ pathway [5]. Inflammatory cytokines and TLR-4 are reported to enhance the activation of NF- $\kappa B$ which contributes in the development of inflammation and apoptosis as it controls several cellular functions [13]. Results of the presented study suggest that treatment with GA attenuates the altered expression of $\mathrm{PI} 3 \mathrm{~K}, \mathrm{NF}-\mathrm{KB}$ and TLR-4 protein the cerebral tissues of ischemic rats.

Several other pathways also contribute in the pathogenesis of neuronal injury in stroke including JAK/STAT/SIRT pathway [21]. It is well documented that in the ischemic condition such as myocardial and cerebral ischemia, JAK/STAT pathway gets activated and further it is also known to be important for the membrane-to-nucleus signalling pathways for a variety of stresses or injuries [3]. Moreover, Sirt-1 is a histone deacetylase which is known for its protective effect against the ischemic condition. A report suggests that alpha-lipoic acid protects the ischemic injury by up-regulation of Sirt-1-dependent PGC-1 $\alpha$ expression [6]. In this investigation, GA treatment ameliorates the altered expression of JAK, STAT, Sirt-1 protein in the neuronal tissue homogenate of cerebral ischemic rats. Moreover, treatment with GA enhances the expression of $\mathrm{BCl}-2$, Akt and PI3K proteins as those have a role in the neuronal apoptosis.

\section{Conclusions}

In conclusion, the results reveal that treatment with Ginkgetin aglycone protects the neuronal injury against stroke in rats. GA is reported to reduce the oxidative stress and inflammation in the brain tissues and also alleviate the altered STAT-3/JAK-2/Sirt-1 pathway. Data of the study suggest that GA could be used clinically for the management of stroke.

\section{Acknowledgements}

All the authors of this manuscript are thankful to Shenyang First Hospital, China for providing the necessary facility to conduct the presented work.

\section{Disclosure}

The authors report no conflict of interest.

\section{References}

1. Akdere $\mathrm{H}$, Tastekin E, Mericliler M, Burgazli KM. The protective effects of Ginkgo biloba EGb761 extract against renal isch- emia-reperfusion injury in rats. Eur Rev Med Pharmacol Sci 2014; 18: 2936-2941.

2. Bhattacharyya A, Chattopadhyay R, Mitra S, Crowe SE. Oxidative stress: an essential factor in the pathogenesis of gastrointestinal mucosal diseases. Physiol Rev 2014; 94: 329-354.

3. Bolli R, Dawn B, Xuan YT. Role of the JAK-STAT pathway in protection against myocardial ischemia/reperfusion injury. Trends Cardiovasc Med 2003; 13: 72-79.

4. Chen H, Yoshioka H, Kim GS, Jung JE, Okami N, Sakata H, Maier CM, Narasimhan P, Goeders CE, Chan PH. Oxidative stress in ischemic brain damage: mechanisms of cell death and potential molecular targets for neuroprotection. Antioxid Redox Signal 2011; 14: 1505-1517.

5. Chen J, Wang Z, Zheng Z, Chen Y, Khor S, Shi K, He Z, Wang Q, Zhao Y, Zhang H, Li X, Li J, Yin J, Wang X, Xiao J. Neuron and microglia/macrophage-derived FGF10 activate neuronal FGFR2/PI3K/Akt signaling and inhibit microglia/macrophages TLR4/NF-кB-dependent neuroinflammation to improve functional recovery after spinal cord injury. Cell Death Dis 2017; 8: e3090.

6. Fu B, Zhang J, Zhang X, Zhang C, Li Y, Zhang Y, He T, Li P, Zhu X, Zhao $Y$, Zhang $Y$, Wang $X$. Alpha-lipoic acid upregulates SIRT1-dependent PGC-1 $\alpha$ expression and protects mouse brain against focal ischemia. Neuroscience 2014; 281: 251-257.

7. Isah T. Rethinking Ginkgo biloba L. Medicinal uses and conservation. Pharmacogn Rev 2015; 9: 140-148.

8. Kalogeris T, Bao Y, Korthuis RJ. Mitochondrial reactive oxygen species: a double edged sword in ischemia/reperfusion vs preconditioning. Redox Biol 2014; 2: 702-714.

9. Kim JY, Kawabori M, Yenari MA. Innate inflammatory responses in stroke: mechanisms and potential therapeutic targets. Curr Med Chem 2014; 21: 2076-2097.

10. Koronowski KB, Perez-Pinzon MA. Sirt1 in cerebral ischemia. Brain Circ 2015; 1: 69-78.

11. Lennicke C, Rahn J, Lichtenfels R, Wessjohann LA, Seliger B. Hydrogen peroxide - production, fate and role in redox signaling of tumor cells. Cell Commun Signal 2015; 13: 39.

12. Li L, Li H, Li M. Curcumin protects against cerebral ischemia-reperfusion injury by activating JAK2/STAT3 signaling pathway in rats. Int J Clin Exp Med 2015; 8: 14985-14991.

13. Liu T, Zhang L, Joo D, Sun SC. NF- $\kappa B$ signaling in inflammation. Signal Transduct Target Ther 2017; 2: 17023.

14. Machado MF, Brucki SM, Nogueira CF, Rocha MS. Infectious disease is the most common cause of death among stroke patients: two-years of follow-up. Arq Neuropsiquiatr 2013; 71 : 371-375.

15. Miao Y, Zhao S, Gao Y, Wang R, Wu Q, Wu H, Luo T. Curcumin pretreatment attenuates inflammation and mitochondrial dysfunction in experimental stroke: The possible role of Sirt1 signaling. Brain Res Bull 2016; 121: 9-15.

16. Naik SR, Pilgaonkar VW, Panda VS. Evaluation of antioxidant activity of Ginkgo biloba phytosomes in rat brain. Phytother Res 2006; 20: 1013-1016.

17. Schaar KL, Brenneman MM, Savitz SI. Functional assessments in the rodent stroke model. Exp Transl Stroke Med 2010; 2: 13. 
18. Shahjouei S, Cai PY, Ansari S, et al. Middle cerebral artery occlusion model of stroke in rodents: a step-by-step approach. J Vasc Interv Neurol 2016; 8: 1-8.

19. Simard JM, Kent TA, Chen M, Tarasov KV, Gerzanich V. Brain oedema in focal ischaemia: molecular pathophysiology and theoretical implications. Lancet Neurol 2007; 6: 258-268.

20. Wu Y, Xu J, Xu J, Zheng W, Chen Q, Jiao D. Study on the mechanism of JAK2/STAT3 signaling pathway-mediated inflammatory reaction after cerebral ischemia. Mol Med Rep 2018; 17: 5007 5012.

21. Zhang F, Wang S, Gan L, Vosler PS, Gao Y, Zigmond MJ, Chen J. Protective effects and mechanisms of sirtuins in the nervous system. Prog Neurobiol 2011; 95: 373-395.

22. Zhang J, Yang S, Chen F, Li H, Chen B. Ginkgetin aglycone ameliorates LPS-induced acute kidney injury by activating SIRT1 via inhibiting the NF-אB signaling pathway. Cell Biosci 2017; 7: 44. 